\title{
Collapsibility of non-cover complexes of graphs
}

\author{
Ilkyoo Choi* \\ Department of Mathematics \\ Hankuk University of Foreign Studies \\ Yongin, Republic of Korea
}

ilkyoo@hufs.ac.kr

\author{
Jinha $\operatorname{Kim}^{\dagger}$ \\ Department of Mathematics \\ Technion - Israel Institute of Technology \\ Haifa, Israel
}

jinhakim@campus.technion.ac.il

Submitted: Apr 23, 2019; Accepted: Dec 22, 2019; Published: Jan 24, 2020

(C) The authors. Released under the CC BY-ND license (International 4.0).

\begin{abstract}
Let $G$ be a graph on the vertex set $V$. A vertex subset $W \subseteq V$ is a cover of $G$ if $V \backslash W$ is an independent set of $G$, and $W$ is a non-cover of $G$ if $W$ is not a cover of $G$. The non-cover complex of $G$ is a simplicial complex on $V$ whose faces are non-covers of $G$. Then the non-cover complex of $G$ is the combinatorial Alexander dual of the independence complex of $G$. Aharoni asked if the non-cover complex of a graph $G$ without isolated vertices is $(|V(G)|-i \gamma(G)-1)$-collapsible where $i \gamma(G)$ denotes the independence domination number of $G$. Extending a result by the second author, who verified Aharoni's question in the affirmative for chordal graphs, we prove that the answer to the question is yes for all graphs.
\end{abstract}

Mathematics Subject Classifications: 05C69, 05E45

*Ilkyoo Choi was supported by the Basic Science Research Program through the National Research Foundation of Korea (NRF) funded by the Ministry of Education (NRF-2018R1D1A1B07043049), and also by the Hankuk University of Foreign Studies Research Fund.

$\dagger$ Corresponding author: jinhakim@campus.technion.ac.il. This research was conducted while Jinha Kim was a graduate student at Seoul National University.

${ }^{\ddagger}$ Boram Park work supported by Basic Science Research Program through the National Research Foundation of Korea (NRF) funded by the Ministry of Science, ICT and Future Planning (NRF2018R1C1B6003577). 


\section{Introduction}

We consider only finite simple graphs. We use the common notation $[n]$ for $\{1, \ldots, n\}$. Given a graph $G$, let $V(G)$ and $E(G)$ denote the vertex set and edge set, respectively, of $G$. An independent set of a graph is a subset of the vertices that induces no edge. A cover of $G$ is a subset $W$ of the vertices such that $V(G) \backslash W$ is an independent set of $G$; in other words, $W$ contains an endpoint of every edge of $G$. A subset of the vertices that is not a cover is called a non-cover.

The independence complex $\mathcal{I}(G)$ of $G$ is a simplicial complex defined as

$$
\mathcal{I}(G):=\{I \subseteq V(G): I \text { is an independent set of } G\},
$$

and the non-cover complex $\mathcal{N C}(G)$ of $G$, which is a simplicial complex defined as

$$
\mathcal{N C}(G):=\{W \subseteq V(G): W \text { is a non-cover of } G\} .
$$

These two simplicial complexes are highly related, in the sense that the non-cover complex $\mathcal{N C}(G)$ is the (combinatorial) Alexander dual of $\mathcal{I}(G)$, where the Alexander dual $D(X)$ of a simplicial complex $X$ on $V$ is defined as

$$
D(X):=\{W \subseteq V: V \backslash W \notin X\}
$$

Note that the non-cover complex of a graph with no edges is the void complex. If a graph with an isolated vertex $v$ has an edge, then the non-cover complex is a cone with apex $v$, and thus it is contractible. However, it is not easy to determine the non-cover complex of an arbitrary graph. Our main result connects the collapsibility of the noncover complex and the independence domination number of the associated graph. We now introduce these two parameters.

For a graph $G$ and $A, D \subseteq V(G)$, if each $v \in A$ has a neighbor in $D$, then we say $D$ dominates $A$. We use $\gamma(G ; A)$ to denote the minimum size of a set that dominates $A$. The independence domination number $i \gamma(G)$ of $G$ is defined as

$$
i \gamma(G):=\max \{\gamma(G ; I): I \in \mathcal{I}(G)\} .
$$

By convention, we let $i \gamma(G)=\infty$ when $G$ contains an isolated vertex.

For a finite simplicial complex $X$, a face $\sigma \in X$ is free if there is a unique facet of $X$ containing $\sigma$. An elementary d-collapse of $X$ is the operation of deleting all faces containing a free face of size at most $d$. We say $X$ is $d$-collapsible if we can obtain the void complex from $X$ by a finite sequence of elementary $d$-collapses. The notion of $d$ collapsibility of simplicial complexes was introduced in [16] and has been widely studied ever since $[11,12]$. An easy observation is that an elementary $d$-collapse does not affect the (non-)vanishing property of homology groups of dimension at least $d$. See also $[7,8]$ for applications regarding Helly-type theorems. In addition, the topological colorful Helly theorem [8] tells us that given a graph $G$ with a $d$-collapsible non-cover complex, for every $d+1$ covers $W_{1}, \ldots, W_{d+1}$ of $G$, there is a cover $W=\left\{w_{i_{1}}, \ldots, w_{i_{k}}\right\}$ of $G$ such that 
$1 \leqslant i_{1}<\cdots<i_{k} \leqslant d+1$ and $w_{i_{j}} \in W_{i_{j}}$ for each $j \in[k]$; the set $W$ is called a rainbow cover of $G$ for $W_{1}, \ldots, W_{d+1}$.

The collapsibility of non-cover complexes of graphs is related to the topological connectivity of independence complexes. For a simplicial complex $X$, let $\eta(X)$ be the maximum integer $k$ such that $\tilde{H}_{j}(X)=0$ for all $-1 \leqslant j \leqslant k-2$. (We use $\tilde{H}_{i}(X)$ to denote the $i$ th reduced homology group of $X$ over $\mathbb{Q}$.) Here, $\tilde{H}_{-1}(X)=0$ if and only if $X$ is non-empty. In $[2,3]$ (see also $[13,14]$ ), it was shown that large independence domination numbers of graphs gives high connectivity of the independence complexes of graphs, in particular, Theorem 1. Research in this direction was motivated by a topological version of Hall's marriage theorem [2].

Theorem 1 ([2,3]). For every graph $G, \eta(\mathcal{I}(G)) \geqslant i \gamma(G)$.

As a consequence of Theorem 1 and the Alexander duality theorem ${ }^{1}$ (see $[6,15]$ ) we obtain that for every graph $G$ with at least one edge, the reduced homology group of the non-cover complex of $G$ satisfies

$$
\tilde{H}_{i}(\mathcal{N C}(G))=0 \text { for all } i \geqslant|V(G)|-i \gamma(G)-1 .
$$

Aharoni [1] asked the following question:

Question 2 ([1]). If $G$ is a graph with no isolated vertices, then is it true that the non-cover complex of $G$ is $(|V(G)|-i \gamma(G)-1)$-collapsible?

The verification of Question 2 for all graphs implies not only the property in (1), but also the stronger property that for every $W \subseteq V(G)$, the reduced homology group of the subcomplex $\mathcal{N C}(G)[W]$ induced by $W$ satisfies

$$
\tilde{H}_{i}(\mathcal{N C}(G)[W])=0 \text { for all } i \geqslant|V(G)|-i \gamma(G)-1 .
$$

In [10], the second author of this paper verified Question 2 for chordal graphs. We extend this result by resolving Question 2 completely in the affirmative.

Theorem 3. For a graph $G$ without isolated vertices, the non-cover complex of $G$ is $(|V(G)|-i \gamma(G)-1)$-collapsible.

The main tool for our proof of Theorem 3 is minimal exclusion sequences [12] (see also [11]), which we review in section 2 along with the proof of Theorem 3 . We end the paper by providing some remarks in section 3 .

\footnotetext{
${ }^{1}$ Alexander duality theorem( $\left.[6,15]\right)$ Let $X$ be a simplicial complex on the vertex set $V$. If $V \notin X$, then for all $-1 \leqslant i \leqslant|V|-2, \tilde{H}_{i}(D(X)) \cong \tilde{H}_{|V|-i-3}(X)$.
} 


\section{Proof}

\subsection{Minimal exclusion sequences}

In this subsection, we review a result in [12], which will play a key role in the proof.

For a simplicial complex $X$ on the vertex set $[n]$, take a linear ordering $\prec_{F}: \sigma_{1}, \ldots, \sigma_{m}$ of the facets of $X$. Given a face $\sigma$ of $X$, we define the minimal exclusion sequence mes $_{\prec_{F}}(\sigma)$ as follows. Let $i$ denote the smallest index such that $\sigma \subseteq \sigma_{i}$. If $i=1$, then $\operatorname{mes}_{\prec_{F}}(\sigma)$ is the null sequence. If $i \geqslant 2$, then $\operatorname{mes}_{\prec_{F}}(\sigma)=\left(v_{1}, \ldots, v_{i-1}\right)$ is a finite sequence of length $i-1$ such that $v_{1}=\min \left(\sigma \backslash \sigma_{1}\right)$ and for each $k \in\{2, \ldots, i-1\}$,

$$
v_{k}= \begin{cases}\min \left(\left\{v_{1}, \ldots, v_{k-1}\right\} \cap\left(\sigma \backslash \sigma_{k}\right)\right) & \text { if }\left\{v_{1}, \ldots, v_{k-1}\right\} \cap\left(\sigma \backslash \sigma_{k}\right) \neq \emptyset \\ \min \left(\sigma \backslash \sigma_{k}\right) & \text { otherwise }\end{cases}
$$

Let $M_{\prec_{F}}(\sigma)$ denote the set of vertices appearing in $\operatorname{mes}_{\prec_{F}}(\sigma)$, and define

$$
d_{\prec_{F}}(X):=\max _{\sigma \in X}\left|M_{\prec_{F}}(\sigma)\right| \text {. }
$$

The following was proved in [12] (see also [11]).

Theorem 4 ([12]). If $\prec_{F}$ is a linear ordering of the facets of $X$, then $X$ is $d_{\prec_{F}}(X)$ collapsible.

\subsection{Proof of Theorem 3}

Let $G$ be a graph without isolated vertices. For simplicity, assume $V(G)=[n]$ and denote $\bar{S}:=[n] \backslash S$ for $S \subseteq[n]$. Let $I$ be an independent set of $G$ such that $\gamma(G ; I)=i \gamma(G)$. Let $|I|=i$. We may assume that $I$ is a maximal independent set and $I:=[n] \backslash[n-i]$.

Note that every facet of $\mathcal{N C}(G)$ is the complement of an edge of $G$. We define a linear ordering $\prec_{F}$ of the facets of $\mathcal{N C}(G)$ as follows. For two edges $a_{1} b_{1}$ and $a_{2} b_{2}$ where $a_{i}<b_{i}$ for $i \in[2]$, let $<_{A L}$ be the anti-lexicographic ordering of $<$, that is, $a_{1} b_{1}<_{A L} a_{2} b_{2}$ if either (i) $b_{1}<b_{2}$ or (ii) $b_{1}=b_{2}$ and $a_{1}<a_{2}$. For two distinct facets $\sigma$ and $\tau$ of $\mathcal{N C}(G)$, we denote $\sigma \prec_{F} \tau$ if $\bar{\sigma}<_{A L} \bar{\tau}$.

Claim 5. For $\sigma, \sigma^{\prime} \in \mathcal{N C}(G)$, if $\bar{\sigma} \cap \bar{I}=\overline{\sigma^{\prime}} \cap \bar{I}$ and $G[\bar{\sigma} \cap \bar{I}]$ contains an edge, then $\operatorname{mes}_{\prec_{F}}(\sigma)=\operatorname{mes}_{\prec_{F}}\left(\sigma^{\prime}\right)$.

Proof. Let $j$ be the length of $\operatorname{mes}_{\prec_{F}}(\sigma)$. Note that an edge between $I$ and $\bar{I}$ comes after all the edges of $G[\bar{I}]$ in the linear ordering $<_{A L}$. Since $G[\bar{\sigma} \cap \bar{I}]$ has an edge, for the $(j+1)$ th facet $\sigma_{j+1}, \overline{\sigma_{j+1}}$ is an edge such that $\overline{\sigma_{j+1}} \subseteq \bar{I}$. By the definition of $\prec_{F}$, it also follows that for every $k \in[j+1]$, the $k$ th facet $\sigma_{k}$ satisfies $\overline{\sigma_{k}} \subseteq \bar{I}$. Clearly, $\sigma \cap \bar{I}=\sigma^{\prime} \cap \bar{I}$. Thus, we have

$$
\overline{\sigma_{k}} \cap \sigma=\overline{\sigma_{k}} \cap \sigma \cap \bar{I}=\overline{\sigma_{k}} \cap \sigma^{\prime} \cap \bar{I}=\overline{\sigma_{k}} \cap \sigma^{\prime} .
$$

Thus the length of $\operatorname{mes}_{\prec_{F}}\left(\sigma^{\prime}\right)$ is also $j$ and for every $k \in[j]$, the $k$ th entry of $\operatorname{mes}_{\prec_{F}}(\sigma)$ is equal to that of $\operatorname{mes}_{\prec_{F}}\left(\sigma^{\prime}\right)$. 
Claim 6. For every $S \subseteq \bar{I}$,

$$
|S|-|N(S) \cap I| \geqslant i \gamma(G)-|I|
$$

where $N(S)=\{v \in V(G): u v \in E(G)$ for some $u \in S\}$.

Proof. Since $G$ has no isolated vertex, for each $v \in I \backslash(N(S) \cap I)$, we can take a neighbor $u_{v} \in \bar{I} \backslash S$ of $v$. Let $T=\left\{u_{v}: v \in I \backslash(N(S) \cap I)\right\}$. Note that $|T| \leqslant|I|-|N(S) \cap I|$ and $S \cup T$ dominates $I$. Thus we obtain

$$
|S|+|I|-|N(S) \cap I| \geqslant|S|+|T| \geqslant i \gamma(G) .
$$

By Theorem 4, it is sufficient to show that

$$
\left|M_{\prec_{F}}(\sigma)\right| \leqslant|V(G)|-i \gamma(G)-1 \quad \text { for every } \sigma \in \mathcal{N C}(G) .
$$

For a face $\sigma \in \mathcal{N C}(G)$, let $\beta(\sigma)=|N(\bar{\sigma} \cap \bar{I}) \cap \bar{\sigma} \cap I|$. Suppose that $\beta(\sigma)=0$. Then $G[\bar{\sigma} \cap \bar{I}]$ must have an edge. Consider $\sigma^{\prime}=\sigma \cap \bar{I}$. Then $\bar{\sigma} \cap \bar{I}=\overline{\sigma^{\prime}} \cap \bar{I}$. By Claim 5 , mes $_{\prec_{F}}(\sigma)=\operatorname{mes}_{\prec_{F}}\left(\sigma^{\prime}\right)$ and therefore, $M_{\prec_{F}}(\sigma)=M_{\prec_{F}}\left(\sigma^{\prime}\right)$. On the other hand, we know $\beta\left(\sigma^{\prime}\right) \geqslant 1$ by the definition of $\sigma^{\prime}$. Thus, it is sufficient to check (2) under the assumption $\beta(\sigma) \geqslant 1$.

We claim that for $v \in \sigma \cap I$, if $v \in M_{\prec_{F}}(\sigma)$, then $v$ is a neighbor of some vertex in $\bar{\sigma} \cap \bar{I}$. Let $k$ be the first index such that the $k$ th entry of $\operatorname{mes}_{\prec_{F}}(\sigma)$ is $v$. Then $v \in \sigma \backslash \sigma_{k}$, which means that $v$ is in the edge $\overline{\sigma_{k}}$. Let $\overline{\sigma_{k}}=w v$ for some vertex $w \in \bar{I}$. Since $w<v$ and $v$ is the $k$ th entry of $\operatorname{mes}_{\prec_{F}}(\sigma)$, we obtain $w \notin \sigma$. Thus $v$ is a neighbor of $w \in \bar{\sigma} \cap \bar{I}$.

Thus,

$$
\begin{aligned}
\left|M_{\prec_{F}}(\sigma)\right| & \leqslant|\sigma \cap \bar{I}|+|N(\bar{\sigma} \cap \bar{I}) \cap(\sigma \cap I)| \\
& =|\bar{I}|-|\bar{\sigma} \cap \bar{I}|+|N(\bar{\sigma} \cap \bar{I}) \cap I|-\beta(\sigma) \\
& \leqslant|\bar{I}|-i \gamma(G)+|I|-\beta(\sigma) \\
& =|V(G)|-i \gamma(G)-\beta(\sigma),
\end{aligned}
$$

where the last inequality holds by applying Claim 6 to the set $\bar{\sigma} \cap \bar{I}$. As we assumed that $\beta(\sigma) \geqslant 1,(2)$ follows, and this concludes the proof of Theorem 3 .

\section{Concluding remarks}

For a graph $G$ and $A, W \subseteq V(G)$, if each $w \in A$ has a neighbor in $W$ or $w \in W$, then we say $W$ weakly dominates $A$. We use $\gamma_{w}(G ; A)$ to denote the minimum size of a set that weakly dominates $A$. The weak independence domination number $i \gamma_{w}(G)$ of $G$ is defined as

$$
i \gamma_{w}(G):=\max \left\{\gamma_{w}(G ; I): I \text { is an independent set of } G\right\} .
$$

The following is a straightforward application of Theorem 3. 
Corollary 7. For a graph $G$, the non-cover complex of $G$ is $\left(|V(G)|-i \gamma_{w}(G)-1\right)$ collapsible.

Proof. If $G$ has no isolated vertex, then $i \gamma_{w}(G)=i \gamma(G)$ and we are done by Theorem 3 . Assume $G$ has $k$ isolated vertices for some integer $k \geqslant 1$. Let $W$ be the set of isolated vertices of $G$, and let $G^{\prime}$ be the graph obtained from $G$ by removing all vertices in $W$.

Recall that $\mathcal{N C}(G)$ is a cone with apex $v$ if $v$ is an isolated vertex of $G$. Thus $\mathcal{N C}(G)$ is $d$-collapsible if and only if the subcomplex of $\mathcal{N C}(G)$ induced by $V(G) \backslash\{v\}$ is $d$-collapsible. Moreover, since the subcomplex of $\mathcal{N C}(G)$ induced by $V(G) \backslash W$ is equal to $\mathcal{N C}\left(G^{\prime}\right)$, it follows that $\mathcal{N C}(G)$ is $d$-collapsible if and only if $\mathcal{N C}\left(G^{\prime}\right)$ is $d$-collapsible. Thus, it is sufficient to show $\mathcal{N C}\left(G^{\prime}\right)$ is $\left(|V(G)|-i \gamma_{w}(G)-1\right)$-collapsible. By Theorem $3, \mathcal{N C}\left(G^{\prime}\right)$ is $\left(\left|V\left(G^{\prime}\right)\right|-i \gamma\left(G^{\prime}\right)-1\right)$-collapsible. Since $\left|V\left(G^{\prime}\right)\right|=|V(G)|-k$ and $i \gamma_{w}(G)=i \gamma\left(G^{\prime}\right)+k$, we obtain $\left|V\left(G^{\prime}\right)\right|-i \gamma\left(G^{\prime}\right)-1=|V(G)|-i \gamma_{w}(G)-1$.

We finish the section by stating a direct consequence of the topological colorful Helly theorem [8] from our main result.

Corollary 8. Let $G$ be a graph on $n$ vertices and let $W_{1}, \ldots, W_{n-i \gamma(G)} \subseteq V(G)$. Assume that every set $A \subseteq V(G)$ satisfying the following two conditions is a cover of $G$ :

(i) $A \cap W_{i} \neq \emptyset$ for $i \in[n-i \gamma(G)]$.

(ii) $W_{j} \subseteq A$ for some $j \in[n-i \gamma(G)]$.

Then there is a cover $W$ of $G$ where $W=\left\{w_{i_{1}}, \ldots, w_{i_{k}}\right\}$ with $1 \leqslant i_{1}<\cdots<i_{k} \leqslant n-i \gamma(G)$ and $w_{i_{j}} \in W_{i_{j}}$ for each $j \in[k]$.

Dao and Schweig [4] showed a weaker version of Theorem 3 concerning a topological property known as "Lerayness" via an algebraic approach. Let us briefly introduce their result. For a simplicial complex $X$, we say $X$ is $d$-Leray if $\tilde{H}_{i}(Y)=0$ for all induced subcomplexes $Y$ of $X$ and all integers $i \geqslant d$. Wegner showed that $d$-collapsiblity implies $d$-Lerayness [16], yet the converse is not always true [12]. Hochster [5] proved the relation between the Leray number ${ }^{2}$ and the Castelnuovo-Mumford regularity of the StanleyReisner ideal of a simplicial complex. From this relationship and the result in [4], it was shown that for a graph $G$, the non-cover complex $\mathcal{N C}(G)$ is $(|V(G)|-i \gamma(G)-1)$-Leray. There is an active line of research in this direction, see $[9,17]$ for more details. By applying the topological colorful Helly theorem of the Lerayness version, we obtain the following:

Corollary 9. Let $G$ be a graph on $n$ vertices. For every $n-i \gamma(G)$ covers $W_{1}, \ldots, W_{n-i \gamma(G)}$ of $G$, there is a cover $W$ of $G$ where $W=\left\{w_{i_{1}}, \ldots, w_{i_{k}}\right\}$ with $1 \leqslant i_{1}<\cdots<i_{k} \leqslant n-i \gamma(G)$ and $w_{i_{j}} \in W_{i_{j}}$ for each $j \in[k]$.

Note that Corollary 9 is weaker than Corollary 8, since if we have $n-i \gamma(G)$ covers for a graph $G$, then a set $A \subseteq V(G)$ satisfying (ii) is a cover of $G$. As mentioned in the introduction, the set $W$ in Corollary 8 and 9 is also known as a rainbow cover of $G$ for $W_{1}, \ldots, W_{n-i \gamma(G)}$. The following example demonstrates that Corollaries 8 and 9 are tight.

\footnotetext{
${ }^{2}$ For a simplicial complex $X$, the Leray number of $X$ is the minimum integer $k$ such that $X$ is $k$-Leray.
} 
Example 10. Let $C_{3 k}$ be a cycle of length $3 k$ for an integer $k \geqslant 2$. It is easy to verify $i \gamma\left(C_{3 k}\right)=k$ and so $\left|V\left(C_{3 k}\right)\right|-i \gamma\left(C_{3 k}\right)=2 k$. Consider $M \subseteq V\left(C_{3 k}\right)$ that induces a matching of size $k$, so that $M$ is a cover of $C_{3 k}$. Let $W_{i}=M$ for all $i \in[2 k-1]$. It is again easy to verify that there is no rainbow cover with respect to $W_{1}, \ldots, W_{2 k-1}$.

\section{Acknowledgements}

The authors thank professor Ron Aharoni for introducing the problem to the second author. They also thank the anonymous reviewer for helpful comments. This work was done during the 4th Korean Early Career Researcher Workshop in Combinatorics.

\section{References}

[1] R. Aharoni. personal communication.

[2] R. Aharoni and P. Haxell. Hall's theorem for hypergraphs. J. Graph Theory, 35(2):83$88,2000$.

[3] M. Chudnovsky. Systems of disjoint representatives. M.Sc. Thesis, Technion, Haifa, 2000.

[4] H. Dao and J. Schweig. Projective dimension, graph domination parameters, and independence complex homology. J. Combin. Theory Ser. A, 120(2):453-469, 2013.

[5] M. Hochster. Cohen-Macaulay rings, combinatorics, and simplicial complexes, in Ring theory, II. Lecture Notes in Pure and Appl. Math., 26:171-223, 1977.

[6] G. Kalai. Enumeration of Q-acyclic simplicial complexes. Israel J. Math., 45:337-351, 1983.

[7] G. Kalai. Intersection patterns of convex sets. Israel J. Math., 48(2-3):161-174, 1984.

[8] G. Kalai and R. Meshulam. A topological colorful Helly theorem. Adv. Math., 191(2):305-311, 2005.

[9] G. Kalai and R. Meshulam. Intersections of Leray complexes and regularity of monomial ideals. J. Combin. Theory Ser. A, 113(7):1586-1592, 2006.

[10] J. Kim. Collapsibility of noncover complexes of chordal graphs. arXiv: 1904.04519, Apr 2019.

[11] A. Lew. Collapsibility of simplicial complexes of hypergraphs. Electron. J. Combin., 26(4):\#P4.10, 2019.

[12] J. Matoušek and M. Tancer. Dimension gaps between representability and collapsibility. Discrete Comput. Geom., 42(4):631-639, 2009.

[13] R. Meshulam. The clique complex and hypergraph matching. Combinatorica, 21(1):89-94, 2001.

[14] R. Meshulam. Domination numbers and homology. J. Combin. Theory Ser. A, 102(2):321-330, 2003. 
[15] R. P. Stanley. Linear diophantine equations and local cohomology. Inv. math., 68:175-193, 1982.

[16] G. Wegner. d-collapsing and nerves of families of convex sets. Arch. Math. (Basel), 26:317-321, 1975.

[17] R. Woodroofe. Matchings, coverings, and Castelnuovo-Mumford regularity. J. Commut. Algebra, 6(2):287-304, 2014. 